references if a deeper understanding is desired, both in the form of chapter bibliographies and a general literature overview at the end of the book. Throughout, the emphasis is on practicality, real problems and working systems. The chapter on catalyst preparation and manufacture (avoided in most books) is a reminder of the multitudinous problems that can be built into a catalyst before measurements or tests are made on it - a sobering reminder that a knowledge of the preparation details is of ten essential to a complete interpretation of catalyst evaluation data.

The second part of the book surveys areas of industrially important catalytic chemistry, with chapters on supported metal catalysts, acid and zeolite catalysts, catalytic oxidation, processing of petroleum hydrocarbons, and synthesis gas and associated processes. These chapters trace the development of process catalysts up to present practice, focussing on details of catalyst composition and process conditions. The chapter on synthesis gas is particularly timely in view of the renewed interest in $\mathrm{CO}$ and $\mathrm{H}_{2}$ as sources of synthetic chemicals, and includes discussions of steam reforming, FischerTropsch synthesis, water-gas shift reactions, methanation, methanol synthesis and ammonia synthesis. Details are generally accurate.

The final chapter on experimental methods covers the engineering details of reactor design, heat and mass transfer, and various types of reactors useful in the laboratory. This is useful to the chemist in that it is reminder of many important noncatalyst effects on experimental results.

The book is eminently readable, in the sense that the author gets to the point in clear prose, and moves on to the next subject. In addition to use in courses in catalysis, the book will provide a valuable review of the subject for the experienced worker in the field, in that its broad coverage of pratical catalyst systems makes it a good initial reference for catalysts and process conditions.

D.E.W. Vaughan is a Research Associate with the Exxon Research and Engineering Company, working in the areas of sorbent and catalytic materials.

\title{
Interactions of binary stars
}

\section{R. J. Tayler}

Close Binary Stars: Observation and Interpretation. Edited by M. J. Plavec and R. K. Ulrich. Pp.598. (Reidel: 1980.) Hardback Dfl.130, \$68.50; paperback Dfl.60, \$31.50.

BINARY stars have always been important for astronomers because the only direct way of determining stellar masses involves the application of Kepler's laws to the observed motions of the components. In addition, a study of eclipsing binaries can give information about stellar radii and variation of brightness across a stellar disk. It has, however, become clear only fairly recently that close binary stars are involved in some of the most violent and exotic phenomena in the Universe.

These phenomena are associated with mass transfer between the components. Most stars increase in size during their life history and a member of a close binary may become so large that its surface layers are attracted to the companion star; this is called Roche lobe overflow. In addition, some stars lose mass continuously and some of this may be captured by the companion. This exchange can have important effects on the evolution of the system. If the initially more massive star becomes the less massive, an ill-matched binary can result in which it appears that the lower mass star has evolved more rapidly than the more massive, in contradiction to basic ideas about stellar evolution. The most dramatic effects occur when one of the component stars is a highly compact star, a white dwarf, a neutron star or a black hole, when a very large release of gravitational energy accompanies mass transfer. This process is important in many $X$-ray sources, novae and cataclysmic variables.

This book is the proceedings of a symposium held in Toronto in the summer of 1979. It contains over a hundred contributions and there are few papers which contain as many as ten pages. As a result, the non-specialist may have some difficulty in discovering what are the key questions and which are the most important papers. I am sorry that there is not a small number of lengthy reviews, since it is clear that there are some important unsolved problems which merit fuller discussion than they receive here. For example, the manner in which close binary systems are formed is not clear and there are some particularly difficult problems and sharp controversies relating to the structure of binaries that are so close that they really form a single star with two nuclei. Further, most calculations of stellar evolution involving mass transfer have assumed conservation of mass and angular momentum by the system, whereas an uncertain amount of each will escape.

Although close binaries are of wide general interest to astronomers, this book is most suitable for those who are already working in the subject.

R. J. Tayler is Professor of Astronomy at the University of Sussex, author of three elementary astronomy textbooks and Managing Editor of Monthly Notices of the Royal Astronomical Society.

\section{Andean archaeology}

\section{John Coles}

Guitarrero Cave: Early Man in the Andes.

Edited by Thomas F. Lynch. Pp.328. (Academic: 1980.) \$20, £13.60.

FOR some years now it has been an archaeological conceit to speak of interdisciplinary projects as the sole way of comprehending any ancient site, settlement or landscape. In few cases have these concepts been achieved, and instead the archaeological world has been presented with rather feeble attempts to coordinate and integrate the variety of natural scientific techniques that can be brought to bear upon most archaeological sites or areas. In the present book we have just such an effort, one which succeeds in part - but not wholly - in demonstrating the virtues of an interdisciplinary approach.

The cave of Guitarrero lies high $(2580 \mathrm{~m})$ in an intermontane valley bounded by the Cordillera Blanca and C. Negra in Peru. The archaeologist will see the possibilities for an ecological research project, with dramatic seasonal variations in environmental conditions presumably being welcomed by the ancient inhabitants, and indeed this form of project is what was conceived and executed. The site was excavated, and its stratigraphical deposits dated by radiocarbon to a period which on balance seems to fall between $10,000 \mathrm{BP}$ and $7,000 \mathrm{BP}$. The environmental implications and attempted reconstruction of the ancient landscapes are heavily reliant on pollen analyses, and there is relatively little on geomorphological processes which one could conceive of as affecting the landscape to a significant degree. Nonetheless, this initial section of the book sets the scene for the major thrust of the project, the study of the quite extraordinarily abundant remains of plants. This is explored through exhaustive analysis of maize and beans, with essays on the origin and early development of cultivation in highland Peru.

A third section provides a full description of the multifarious stone artifacts from the major stratigraphic phases of the cave occupation, and is somewhat tedious in approach. More interesting by virtue of rarity are the bone and wood artifacts, and remains of cordage, basketry and textiles which conditions in parts of the cave have allowed to survive. The final summary and overview of the site emphasizes this organic content which alone would have made the excavation worthwhile.

Yet, as regards disciplines, multidisciplinary it is, interdisciplinary it is not. Archaeologists may never be able to achieve the latter if they remain content with the former.

John Coles is Professor of European Prehistory at the University of Cambridge. 